\title{
La Galissonière et ses conceptions coloniales d'après le "Mémoire sur les colonies de la France dans l'Amérique septentrionale » (décembre 1750)
}

\section{Roland Lamontagne}

Volume 15, numéro 2, septembre 1961

URI : https://id.erudit.org/iderudit/302109ar

DOI : https://doi.org/10.7202/302109ar

Aller au sommaire du numéro

Éditeur(s)

Institut d'histoire de l'Amérique française

ISSN

0035-2357 (imprimé)

1492-1383 (numérique)

Découvrir la revue

Citer cet article

Lamontagne, R. (1961). La Galissonière et ses conceptions coloniales d'après le

"Mémoire sur les colonies de la France dans l'Amérique septentrionale "

(décembre 1750). Revue d'histoire de l'Amérique française, 15(2), 163-170.

https://doi.org/10.7202/302109ar d'utilisation que vous pouvez consulter en ligne. 


\section{LA GALISSONIËRE ET SES CONCEPTIONS COLONIALES D'APRĖS LE «MÉMOIRE SUR LES COLONIES DE LA FRANCE DANS L'AMÉRIQUE SEPTENTRIONALE 》 (décembre 1750) *}

Louis XV et le roi de Grande-Bretagne, Georges II, avaient décidé de confier à des commissaires la tâche de régler «toutes les prétentions réciproques» concernant les limites de leurs colonies respectives en Amérique du Nord. ${ }^{1}$ Le 24 août 1750, Louis XV accorde les pleins pouvoirs de commissaire du roi à Roland-Michel Barrin de La Galissonière et à Étienne de Silhouette. ${ }^{2}$ William Mildmay et le gouverneur de Massachusetts Bay Colony, William Shirley, représentent le roi de GrandeBretagne. ${ }^{3}$

Selon une note marginale qui n'est pas signée, ${ }^{4}$ La Galissonière et Silhouette ont rédigé le «Mémoire sur les colonies de la France dans l'Amérique septentrionale ». Le volume 24 des archives du Ministère des Affaires Étrangères dans le fonds dit:

* Etude présentée à la Réunion générale de l'Institut d'histoire de l'Amérique française, le 8 avril 1961.

1 Les conclusions d'enquêtes des commissaires des rois britannique et français mettent en relief la complexité de la question de litige concernant les limites territoriales. Mémoires des commissaires du roi et de ceux de Sa Majesté britannique sur les possessions et les droits respectifs des deux couronnes en Amérique avec les actes publics et pièces justificatives (4 vol., Paris, 1755 - 1757).

2 Affaires Etrangères. Correspondance politique. Angleterre, 17501751, vol. 430 - 431. Archives publiques du Canada, M. G. 5, A - 1 (32), 119120.

3 Lawrence Henry Gipson, Zones of International Friction: The Great Lakes Frontier, Canada, The West Indies, India, 1748-1754. The British Empire before the American Revolution (New York, 1939), 5: 303.

4 \& Ce memoire est tel qu'il avoit été redigé Par M.M. Silhouette et de la Galissoniere Mais $\mathrm{M}$. le $\mathrm{Ma}$ ' al de noiailles ayant trouvé qu'on y etendoit trop les inconveniens on la Reduit comme dans la Copie n². » AC, C11A, $96: 175$. 
«Mémoires et Documents, Amérique » n'indique qu'un nom d'auteur, celui de Roland-Michel Barrin de La Galissonière. ${ }^{5}$ D'après un document du 8 février 1759, il semble bien qu'il faille attribuer à La Galissonière le mérite de cette étude de la situation coloniale. Ce document est anonyme; on ne possède aucun indice qui puisse révéler l'identité de son auteur. ${ }^{6}$

En 1751 M. de la Galissoniere recommendable par ses vertus, par ses connoissances et par son zèle pour la gloire du Roi et de l'Etat, présenta un mémoire dont tous les ministres du Roy voulurent avoir une copie, concernant les Colonies de la France dans l'Amérique septentrionale. Il avoit commandé dans le Canada; Il avoit été dans toutes ses Colonies; Il les avoit étudiées et il les connoissoit; Il regardoit le Canada comme le boulevard de toutes les autres colonies...

Cette dernière remarque, légèrement modifiée, est extraite du texte du Mémoire: «on ne doit rien épargner pour mettre ces Colonies en force, puisqu'on peut et qu'on doit les considérer comme le boulevard de l'Amérique contre les entreprises des Anglois .... ${ }^{7}$

Ce mémoire incite la Cour à porter «l'attention la plus sérieuse » aux prétentions qu'ont exprimées les commissaires du roi de la Grande-Bretagne sur l'étendue de l'Acadie. Il a pour but de démontrer qu'il est nécessaire de maintenir l'intégrité des frontières coloniales et de coordonner les éléments de défense militaire. La Galissonière signale l'inquiétude que provoquent les Britanniques en Amérique du Nord: «Tandis que la paix paroit avoir comme assoupi la jalousie des Anglois en Europe, elle éclate dans toute sa force en Amerique; et si on n'y oppose dès apresent des barrieres capables d'en arrêter les effets, cette

5 « Mémoire sur les colonies de la France, dans l'Amérique septentrionale. Par M. le Marquis de la Galissonnière. 》 APC. M.G. 5, B - 1 (22), 180-228.

6 Monsieur Pierre Brunet des Archives publiques du Canada a eu l'obligeance de fournir ce renseignement. Affaires Etrangères. Mémoires et Documents. Amérique, vol. 24, pt. 3, 1518-1759. APC. M.G. 5, B-1 (23), 434.

7 AC, C11A, 96: 211. 
Nation se mettra en état d'envahir entierement les Colonies françoises au commencement de la premiere guerre. $\gg^{8}$

Le cardinal de Bernis a jugé sévèrement la politique française de cette époque: "On a fermé les yeux, dit-il, sur de petits actes d'hostilité commis par nous contre les Anglais en Amérique; nous avons mal à propos élevé des forts qui ont donné de la jalousie; nous avons dépensé beaucoup d'argent pour la défense de la colonie, et cet argent a été mal employé et dissipé. ${ }^{9}$

Le mémoire de décembre 1750 présente les « raisons essentielles et capitales » de veiller avec soin à la conservation, à la consolidation et à l'expansion de la colonie. ${ }^{10}$ Nous verrons que Roland-Michel Barrin de La Galissonière s'appuie sur les principes de colonisation systématique.

La civilisation se définit par les manifestations de la vie religieuse, politique et sociale ainsi que par un ensemble d'institutions d'ordre intellectuel, artistique et scientifique et d'activité économique et technique. La colonisation est corollaire de la civilisation. Si elle s'effectue dans un pays déjà organisé, elle consiste en une entreprise d'exploitation économique. Dans une colonie de peuplement, l'axe de développement s'oriente normalement vers la formation d'une nouvelle patrie à l'image de l'État métropolitain.

A l'aide d'immigrants, d'institutions politiques et de finances publiques et privées, la colonisation met en valeur un territoire en implantant des activités variées; elle assure à la population une vie religieuse, sociale et intellectuelle; elle contribue à l'aménagement d'une structure économique. Une vie économique complète comprend les divers secteurs de l'agriculture, de l'exploitation des matières premières, du commerce, de l'industrie et des finances formées de capitaux de la métropole, de la colonie et de l'entreprise privée. L'utilisation des cours d'eau, du sol et du sous-sol constitue des facteurs importants de colonisation systé-

8 Ibid., 175.

9 Frédéric Masson, éd., Mémoires et lettres de François-Joachim de Pierre cardinal de Bernis, 1715-1758, (2 vol., Paris, 1903), 1: 135. Cité par Guy Frégault, François Bigot, administrateur français, '(2 vol., Montréal, 1948), 2: 70.

$10 \mathrm{AC}, \mathrm{C} 11 \mathrm{~A}, 96: 176$. 
matique. Les principes de colonisation ${ }^{11}$ sont sous-jacents à l'étude d'ensemble de Émile Salone, La Colonisation de la Nouvelle-France.

En raison de l'interprétation historique des mouvements migratoires, des causes d'ordre religieux, politique, économique, psychologique, notamment la richesse des matières premières dans les territoires à coloniser et l'attrait des pays neufs, poussent les êtres humains à émigrer. Le peuplement constitue la condition fondamentale de la colonisation. La Galissonière propose d'accroître la population de la Nouvelle-France. ${ }^{12}$ A maintes reprises, il rappelle la nécessité de l'augmentation démographique pour y rattacher des remarques sur la défense militaire de la colonie. Il insiste sur l'importance stratégique de l'Acadie. Il trouve essentiel de fonder « un gros village françois bien fortifié » dans la région $d u$ fort Saint-Frédéric ${ }^{13}$ ainsi que des postes sur la Belle Rivière [Ohio]. ${ }^{14}$ Il veut peupler la région des Illinois: «il y a déjà quelques habitans fournis d'un assés bon nombre de bestiaux, mais ce n'est rien en comparaison de ce qu'on y pouroit placer $\gg .{ }^{15}$

La Galissonière apporte des précisions sur la nature de ce peuplement qui doit être formé principalement de soldats «dont on poura dans tres peu de tems faire de bons habitans ». Il note que l'on peut y joindre un petit nombre de contrebandiers, de mendiants valides et même quelques femmes de mauvaise vie. Il ajoute avec prudence: «il ne faut envoyer de ces dernières que quand on en demandera, ce qui vraisemblablement ne sera pas sitôt, y ayant en general dans le Canada plus de femmes que d'hommes ».

11 Le lecteur pourrait consulter avec profit les études suivantes: G. Frégault, « Essai sur les finances canadiennes, 1700-1750 》, Revue d'Histoire de l'Amérique française, 13 (1959) : 181; M. Brunet, «Le rôle des métropoles et des entrepreneurs dans la colonisation de l'Amérique et la mise en valeur de la vallée du Saint-Laurent 》. Tiré à part d'une communication présentée lors de la réunion annuelle de La Société Historique du Canada, à Saskatoon (1959).

12 AC, C11A, 96: 211.

13 Ibid., 198.

14 Ibid., 205.

15 Ibid., 206. 
Le mémoire de décembre 1750 encourage le gouvernement de la mère-patrie à organiser un vaste mouvement d'émigration. «On doit se déterminer a envoyer beaucoup de monde a la nouvelle france, afin de mettre ceux qui en ont l'administra'on en état de travailler en meme tems aux differens établissemens proposés. » 16

La Galissonière ne se limite pas à l'aspect de colonisation militaire et agricole. Il s'intéresse à l'effet du peuplement sur l'activité économique: «La Louisiane est un pays tres susceptible de cultures utiles, et auquel il ne manque que des habitans ... Les progrès du commerce seront plus rapides en peuplant la partie a portée de la mer ... ${ }^{17}$ La situation géographique de Détroit est propre à la formation d'une agglomération urbaine.

C'est a ce dernier endroit [Détroit] qu'il faut aujourd'huy le plus s'attacher. S'il y avoit une fois dans ce canton mille habitans cultivateurs, il nourriroit et defendroit tous les autres. C'est de tout l'interieur de Canada, l'endroit le plus propre a établir une ville ou se réuniroit tout le commerce des Lacs et qui munie d'une bonne garnison et entourée d'un bon nombre d'habitations seroit aportée d'en imposer a presque tous les Sauvages du Continent. ${ }^{18}$

Avant d'analyser la situation déficitaire de la colonie, La Galissonière met en relief les avantages qui découlent de la colonisation; il est vrai que ces considérations sur l'utilité des colonies s'inscrivent dans le cadre du mercantilisme. Nous verrons plus loin que La Galissonière cherche à acheminer la société coloniale vers une certaine indépendance par rapport à l'Etat métropolitain.

S'il y avoit encore dans les esprits quelque incertitude a cet égard [l'utilité des colonies], il suffiroit pour la dissiper de jetter les yeux sur les comptes des produits des fermes du Roy, sur la quantité immense de toutes [sortes] de denrées et de manufactures qui passent dans les Colonies, sur les retours qui en proviennent, dont les uns sont nécessaires aux manu-

16 Ibid., 211.

17 Ibid., 209.

18 Ibid., 205. 
factures, et les autres à des consommations devenues habitueles dans le Royaume, et en quelque sorte indispensables: Enfin sur l'excedent qui passe a l'Etranger et qui contribue si essentiellement a faire pancher la balance des richesses en faveur de la france: objets qui deviennent de jour en jour plus interessans a mesure que chaque Etat forme de nouveaux projets pour se passer des productions et des manufactures de ses voisins. ${ }^{19}$

La Galissonière encourage l'établissement d'industries de transformation, notamment des fabriques d'armement, dans la colonie, sous prétexte d'alléger le fardeau des dépenses métropolitaines. ${ }^{20}$ Il veut articuler le développement technique de la région des Illinois à une solide structure démographique et agricole. «On ne parle point des mines qu'on prétend avoir découvert dans ce Canton. Independamment de ce qu'on n'est pas sufisamment informé, on estime qu'on n'y doit penser qu'aprés qu'on se sera fortifié sufisamment, mais principalement en hommes, en bled et en bestiaux. ${ }^{21}$

La Galissonière recommande de protéger l'armature religieuse de la société coloniale. "Les motifs d'honneur, de gloire et de Religion ne permettent point d'abandonner une Colonie établie, de livrer a eux memes ou plustôt a une Nation ennemie par goût, par education et par principe de Religion les françois qui y ont passé a la persuasion du Gouvernement sous l'esperance de sa protection, et qui la meritent singulierement par leur fidelité et leur attachement; Enfin de renoncer a un ouvrage aussi salutaire que celui de la conversion des Infideles qui habitent ce vaste continent. » 22

19 Ibid., 177-178.

20 « On ne doit pas se flatter de pouvoir de longtems soutenir la depense d'une marine égale a la leur [Britanniques]; il ne reste donc que la ressource de les attaquer dans leurs possessions, cela ne peut se faire par des forces envoyées d'Europe qu'avec peu d'espérance de réussir et avec beaucoup de depense, au lieu qu'en se fortiffiant en Amerique, et en se menageant des moyens dans les Colonies memes, on peut se conserver les avantages dont on est en possession, et on peut meme les augmenter avec une dépense trés médiocre en comparaison de celle que couteroient des armemens faits en Europe. »Ibid., 186.

21 Ibid., 208.

22 Ibid., 181-182. 
La Galissonière reconnaît que « les depenses de ces Colonies non seulement surpassent et surpasseront longtems de beaucoup leur produit, mais elles sont fort inégales et sujetes a des augmentations forcées surtout en tems de guerre...». L'État métropolitain pourra éventuellement escompter des profits de ces vastes territoires, des terres fertiles, des forêts, des gisements miniers. Cette remarque est empreinte d'une politique mercantiliste.

Des points d'insistance du mémoire portent sur l'organisation d'un mouvement d'immigration en Nouvelle-France. Le Canada pourrait venir en aide aux autres colonies de la France en Amérique. ${ }^{23}$ La Galissonière conseille de fortifier la NouvelleFrance et de la rendre productive.

Le mémoire de décembre 1750 repose sur les principes de la colonisation tels que le peuplement, le maintien de l'organisation religieuse et politique, l'aménagement de l'agriculture, du commerce et de la technique. L'aspect culturel, conditionné par les facteurs qui viennent d'être énumérés, n'est pas analysé dans ce rapport; la défense militaire forme le centre de préoccupation, elle est reliée à l'essor démographique ainsi qu'à un apport accru de finances métropolitaines. La Galissonière insiste sur l'interdépendance de la France et des colonies:

Les depenses qu'elles [les colonies] occasionneront en épargneront de plus considerables, et beaucoup moins certaines dans leurs effets, surtout si on se laissoit reduire a la nécessité d'y faire passer des secours de france pour les soutenir en tems de guerre; Et qu'on ne peut les abandonner a leurs seules forces actuelles, sans les livrer en quelque sorte aux anglois a qui la superiorité en Amérique, et les richesses qu'ils en retireroient a l'exclusion des autres Nations, donneroient tres certainement la superiorité en Europe. ${ }^{24}$

23 « Le Canada où la fécondité est merveilleuse peut servir de pepiniere pour entretenir les Colonies du Tropique, qui vendent bien cher leurs richesses par les hommes qu'elles consomment. Il est d'expérience qu'il meurt beaucoup moins de Canadiens que de françois Européens dans les endroits de ces Colonies reconnus pour être les plus malsains. 》 Ibid., 187-188.

24 Ibid., 212. 
Le mémoire de décembre 1750 ne constitue pas une étude complète de la colonisation. Il porte la marque de l'expérience, de l'initiative, des conceptions coloniales du politique, du militaire et de l'administrateur aux vues pénétrantes que fut RolandMichel Barrin de La Galissonière.

Roland Lamontagne, Ph.D. (histoire),

Professeur à la Faculté des Lettres de l'Université de Montréal. 